\title{
Imaging in noncardiovascular thoracic emergencies: a pictorial review
}

Ashish Chawla, MD, DABR

\begin{abstract}
Cardiovascular and noncardiovascular conditions are commonly encountered in the emergency department. While the majority of patients have underlying cardiovascular aetiologies, such as acute myocardial infarction, congestive heart failure, aortic dissection and pulmonary embolism, a small subset of patients have underlying noncardiovascular conditions, although they present with similar symptoms of chest pain, dyspnoea, cough, haemoptysis and haematemesis. This article aims to describe the imaging findings in common noncardiovascular conditions of the chest that are frequently encountered in the emergency department, with a review of the existing literature.
\end{abstract}

Keywords: asthma, emergency department, oesophagitis, pneumomediastinum, pneumothorax

\section{INTRODUCTION}

Thoracic emergencies are mainly diagnosed and differentiated through clinical history, physical examination, electrocardiography and laboratory investigations. For a substantial proportion of patients presenting to the emergency department (ED), a specific diagnosis remains elusive and imaging plays an important role in the assessment of patients with cardiac and noncardiac chest conditions. This article aims to discuss the role of radiography and computed tomography (CT) of the chest in evaluating noncardiovascular thoracic diseases encountered in the ED. It illustrates the spectrum of noncardiovascular thoracic emergency conditions and reviews specific imaging features of conditions that are commonly encountered. Although clinical presentations may vary, there are many similarities in presenting symptoms, signs and clinical history across different conditions. Based on the dominant clinical features, acute conditions can be divided into seven categories (Table I).

\section{ROLE OF IMAGING}

\section{Chest pain}

Chest pain is the most common, specific and principal reason for ED visits by adults. ${ }^{(1)}$ Cardiac causes are the most alarming, followed by pulmonary, musculoskeletal and oesophageal causes. Imaging does not play a major role in diagnosing musculoskeletal aetiologies. However, chest radiography and $\mathrm{CT}$ are useful for evaluating chest pains of pleuropulmonary origin, such as pneumothorax, pneumohaemothorax and pneumomediastinum.

Erect chest radiography is sufficient to demonstrate pneumothorax and pneumomediastinum. CT is performed to identify the underlying aetiology, so that proper treatment can be planned to prevent a recurrence. In patients with pneumothorax, CT can reveal underlying blebs, emphysema, cystic lung disease, infection or interstitial lung disease (Fig. 1). Primary spontaneous pneumothorax is seen in patients who are young, tall and thin; the cause is postulated to be the
Table I. Common clinical presentations and noncardiovascular diseases encountered in the emergency department that require radiological investigations.

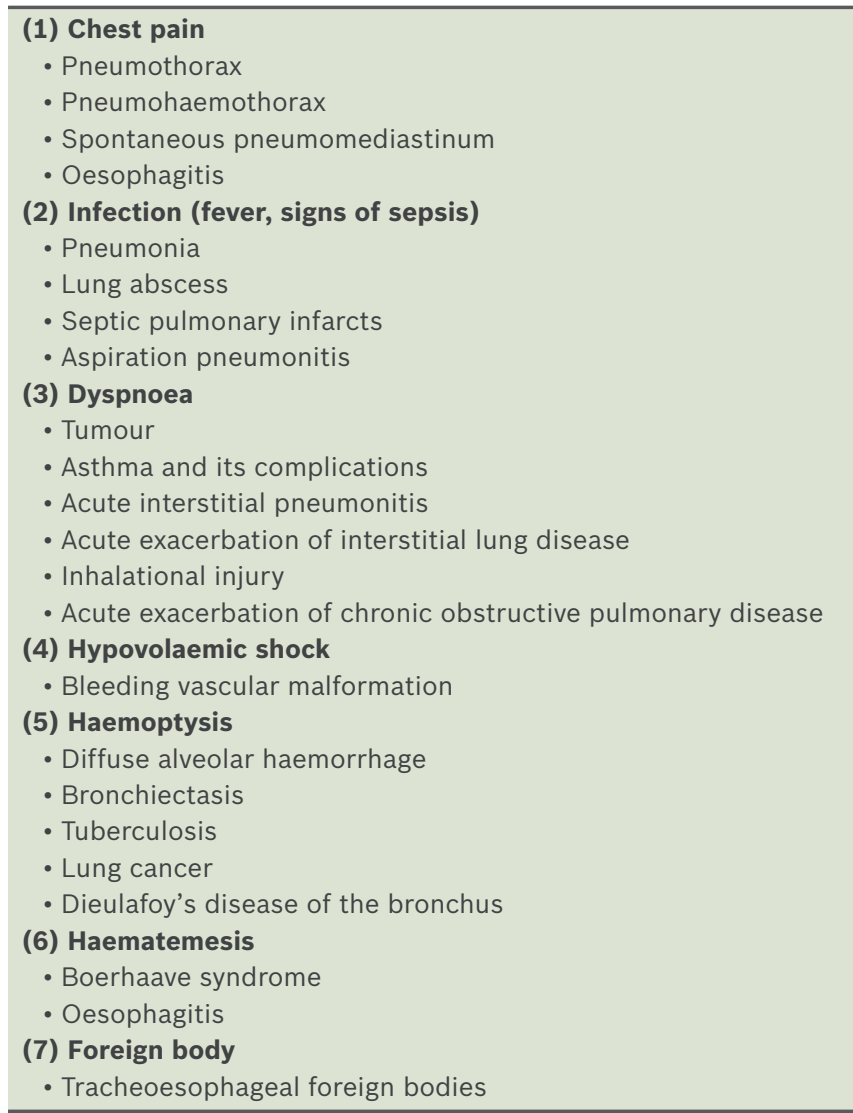

rupture of subpleural blebs. ${ }^{(2)}$ However, blebs are identified on imaging and during surgery in only a small proportion of these patients. ${ }^{(3)}$ Spontaneous haemopneumothorax, in which haemothorax and pneumothorax occur concurrently, is another rare entity observed in men aged $20-40$ years (Fig. 2). Spontaneous haemothorax is a complication in $3 \%-7 \%$ of cases of spontaneous pneumothorax. ${ }^{(4)}$ The postulated mechanisms 

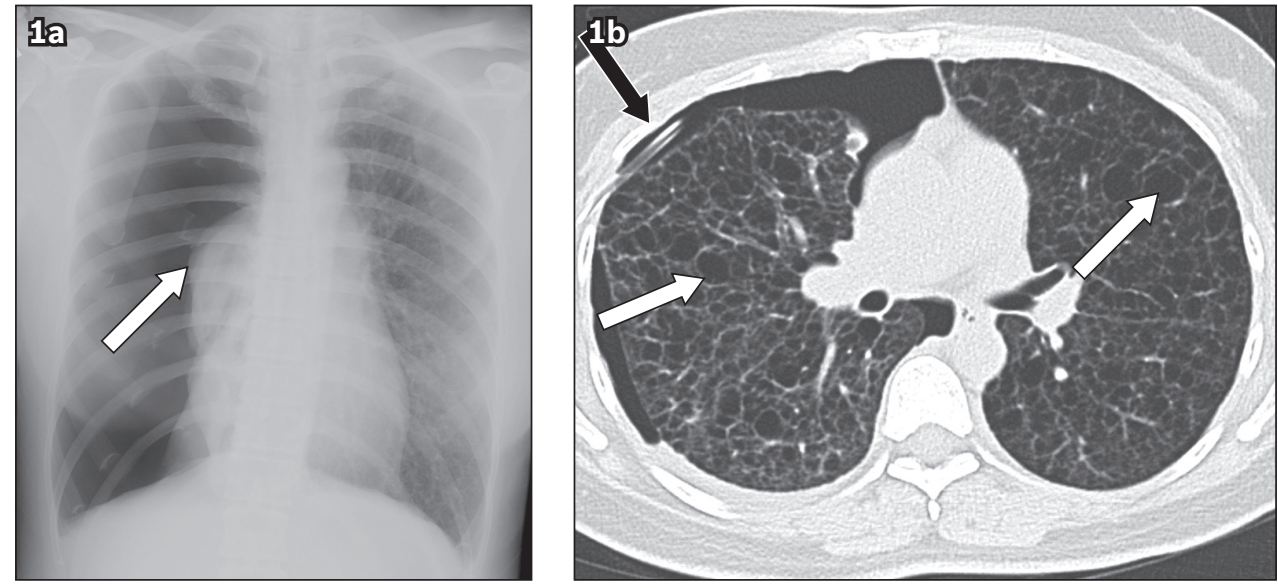

Fig. 1 Large spontaneous pneumothorax in a 40-year-old woman with lymphangioleiomyomatosis. (a) Fronta chest radiograph shows a large right-sided pneumothorax with collapsed lung at the hilum (white arrow). (b) Axial CT image shows numerous cysts in both lungs (white arrows), with a chest tube for drainage of the pneumothorax (black arrow).
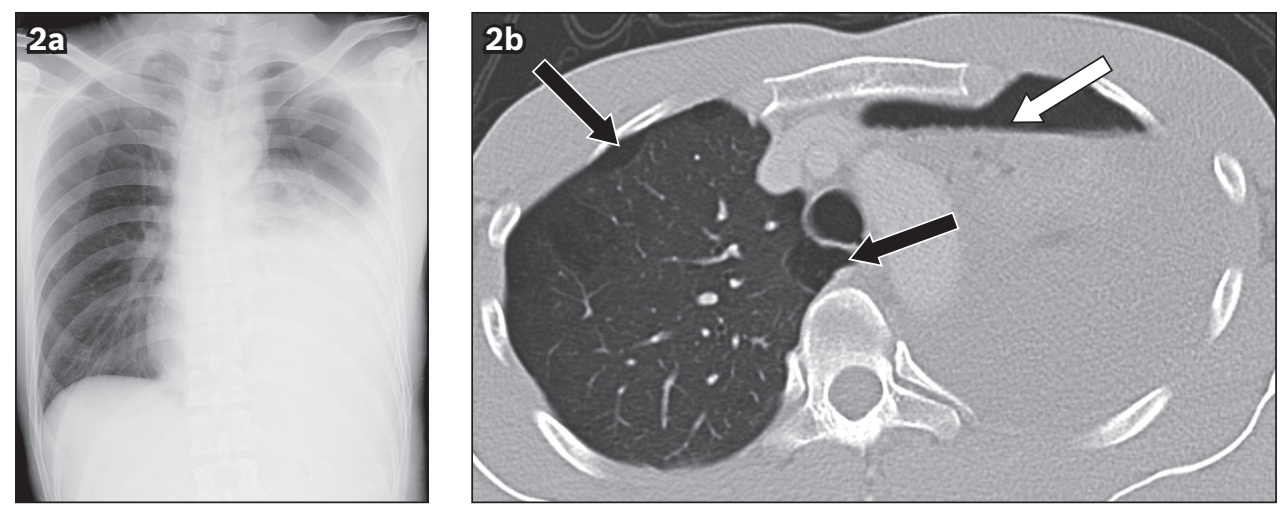

Fig. 2 Spontaneous haemopneumothorax in a 39-year-old man presenting to the emergency department with chest pain and giddiness. (a) Frontal chest radiograph shows a large amount of left-sided pleural fluid, without evidence of pneumothorax. (b) Axial CT image shows an air-fluid level (white arrow) in the left hemithorax with a large complex fluid component. The aerated right lung shows subpleural blebs (black arrows). The drained fluid was dark-coloured, thick and bloodstained. The haemopneumothorax was probably secondary to bleeding in spontaneous pneumothorax from a ruptured vascularised bleb or due to vascular injury.
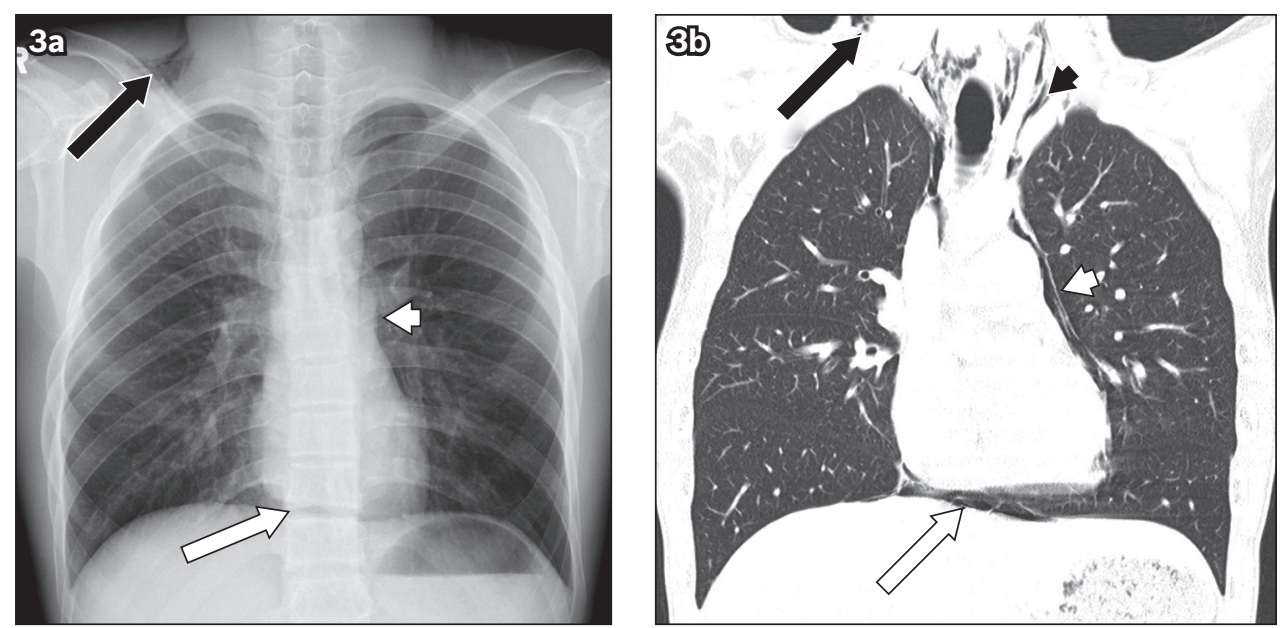

Fig. 3 Spontaneous pneumomediastinum in a 17-year-old boy presenting to the emergency department with sudden onset of excruciating chest pain. (a) Frontal chest radiograph and (b) coronal CT image show paracardiac air (white arrowheads), continuous diaphragm sign (white arrows), and supraclavicular emphysema (black arrows) and emphysema in the superior mediastinum (black arrowhead)

for spontaneous haemopneumothorax are: (a) torn adhesion between the parietal and visceral pleura; (b) tear in a small noncontractile vessel on the parietal pleura; or (c) rupture of a vascularised bulla. ${ }^{(4)}$ Pneumomediastinum usually results from perforation of the oesophagus, tracheobronchial trees or lungs. When no sources of air in the mediastinum are identified on endoscopic studies, CT and barium examinations, a diagnosis of spontaneous pneumomediastinum is considered (Fig. 3). 

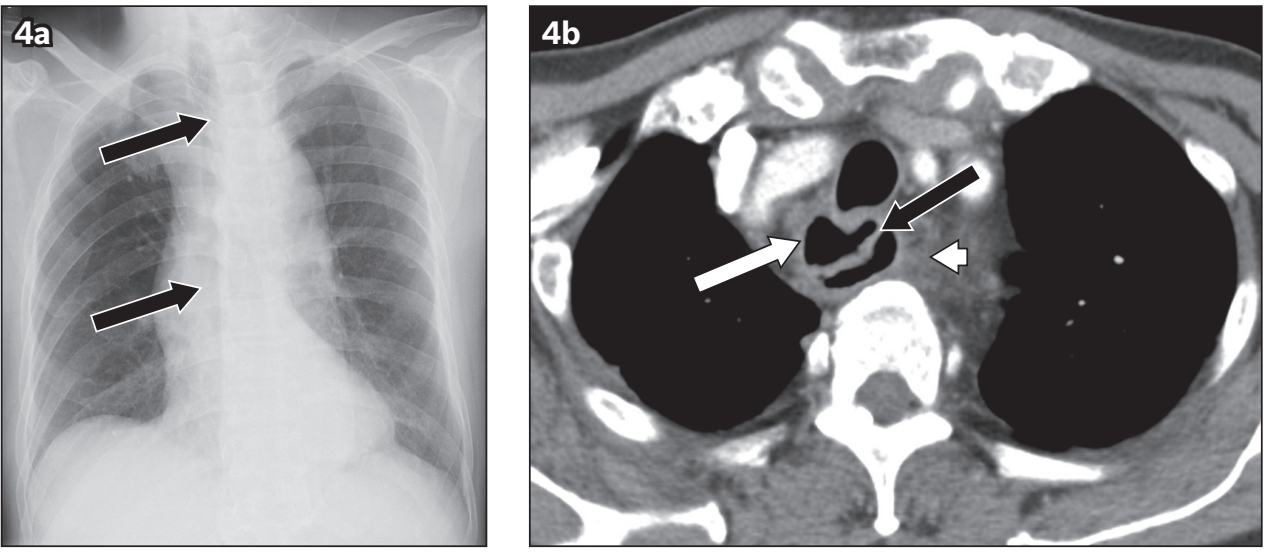

Fig. 4 Severe ulcerative oesophagitis in a 70-year-old man with renal failure. (a) Frontal chest radiograph shows vertical linear lucency (black arrows) in the midline, representing the dilated oesophagus. (b) Axial CT image of the upper oesophagus shows diffuse thickening of the oesophageal wall (white arrow) and an overhanging mucosa (black arrow), creating a 'double lumen', which is associated with fat stranding in the mediastinum (white arrowhead).
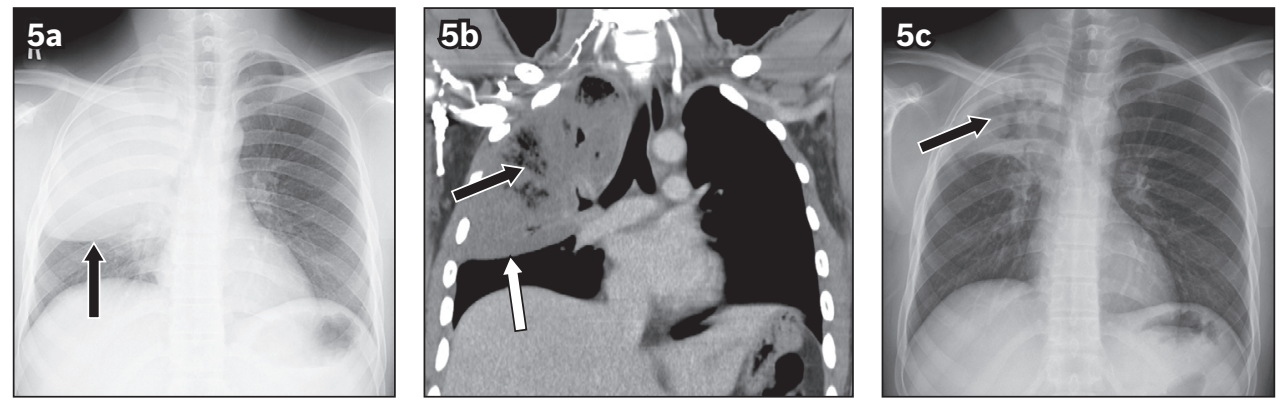

Fig. 5 Klebsiella pneumonia in a 35-year-old man presenting to the emergency department with high-grade fever, rigors, cough and high total white blood cell count. (a) Frontal chest radiograph shows a large, dense homogeneous opacity in the right upper hemithorax, with a sagging inferior border (black arrow). (b) Corresponding coronal CT image shows necrotising consolidation (black arrow) in the right upper lobe with the bulging fissure sign (white arrow). (c) Follow-up chest radiograph after treatment shows residual scarring in the right upper lung (black arrow).

The pathophysiology of spontaneous pneumomediastinum has been attributed to the Macklin effect. ${ }^{(5)}$ Macklin reported that mediastinal emphysema is secondary to tears in the alveolar sacs near the pulmonary ligament. The leaked air then dissects along the bronchovascular sheaths and tracks into the mediastinum. ${ }^{(5)}$

Oesophageal inflammation and gastro-oesophageal reflux disease can lead to chest pain. A moderate-to-large hiatal hernia can be visualised on chest radiography, but it may or may not be the cause of the pain. Life-threatening cardiogenic pain needs to be excluded before the diagnosis of gastro-oesophageal reflux disease can be considered. Infrequently, severe oesophagitis (Fig. 4) can be diagnosed on CT. ${ }^{(6)}$

\section{Infection}

Chest radiography remains the initial investigation of choice for all patients presenting with signs of pneumonia at the ED. Frontal radiography of the chest provides useful information about the extent and severity of the pneumonia and associated pleural effusions. Chest radiography followed by CT can help in identifying the source and aetiology of the pneumonia in a small fraction of patients. In patients with Klebsiella pneumonia, a classical buldging fissure sign (Fig. 5) has been described.(7) Aspiration pneumonia may be suggested on CT and is typically found in the lower dependent lungs, along with a dilated oesophagus. The characteristic appearance of septic pulmonary emboli can also be identified on imaging. In a patient with clinical features of sepsis, multiple peripheral wedge-shaped consolidations, with or without cavitations, suggest the diagnosis of septic pulmonary emboli. ${ }^{(8)}$ CT can also identify other foci of infection (Fig. 6) and/or jugular vein thrombophlebitis, as well as filling defects in the left cardiac chambers. CT is also used to investigate transpleural hepatopulmonary abscesses (Fig. 7), which are almost always due to Entamoeba histolytica in endemic areas. ${ }^{(9)}$

\section{Dyspnoea}

Most patients who present to the ED with dyspnoea as the predominant symptom have an underlying cardiac disease, but a significant proportion of them have an underlying pulmonary pathology. Chest radiography is a useful tool to differentiate between these two subsets of patients. Imaging also plays an important role in diagnosing the most common pulmonary causes of dyspnoea - acute lung collapse (Fig. 8) and acute exacerbation of chronic obstructive pulmonary disease, asthma and interstitial lung disease (ILD). Asthmatic patients mainly present to the ED with acute episodes of asthma. For these patients, the role of imaging is identifying complications such as pneumothorax, pneumomediastinum (Fig. 9), infective pneumonia, eosinophilic 

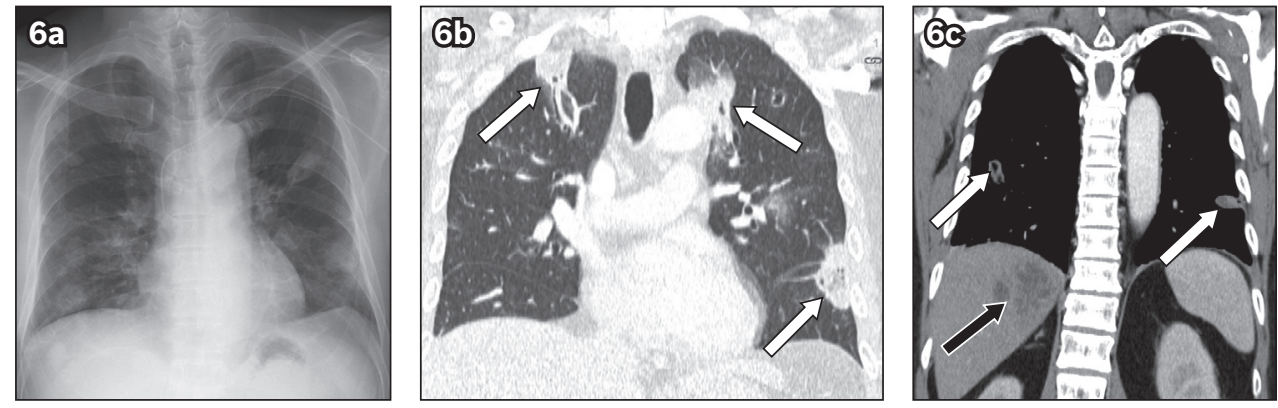

Fig. 6 Septic pulmonary emboli in a 62-year-old man being treated for liver abscess. (a) Frontal chest radiograph shows multiple peripheral wedge-shaped opacities. (b) Coronal CT image shows peripheral pulmonary opacities with cavitations (white arrows). (c) Coronal CT image in soft tissue window shows a left hepatic abscess (black arrow) and cavitary lesions (white arrows).
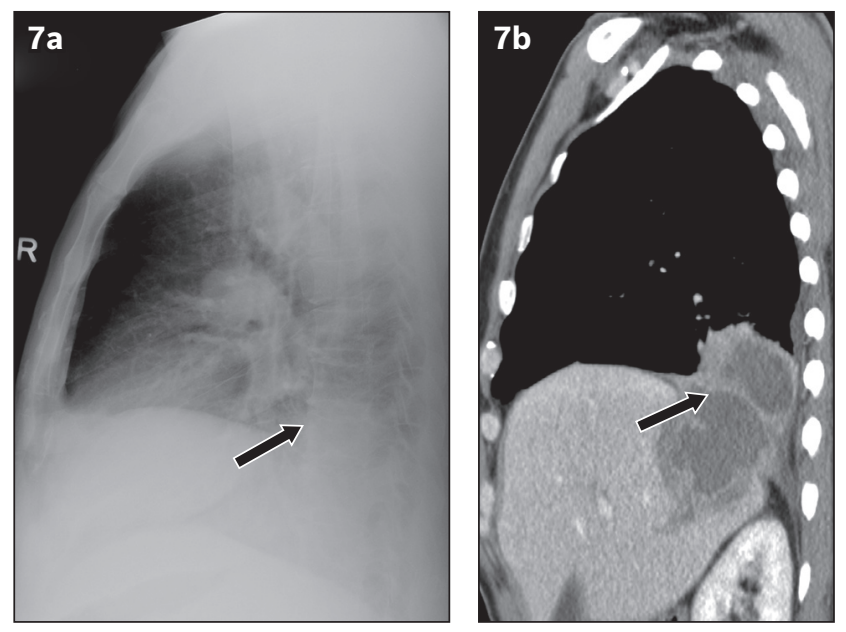

Fig. 7 Amoebic hepatopulmonary abscess in a 55-year-old man presenting to the emergency department with cough and fever. (a) Lateral chest radiograph shows an ill-defined opacity in the posterior lower lung (arrow). (b) Sagittal CT image shows transpleural rupture of the liver abscess into the lower lobe of the left lung (arrow). Only amoebic liver abscesses are known to follow this route in the endemic zone.

pneumonia, allergic bronchopulmonary mycosis and lobar collapse from mucus plugging. ${ }^{(10)}$

Acute interstitial pneumonitis, a rare acute condition that affects an otherwise normal lung, is characterised by progressive dyspnoea and multifocal pulmonary opacities. ${ }^{111}$ Diffuse alveolar damage is the histological hallmark of this condition. ${ }^{122}$ Acute interstitial pneumonitis worsens rapidly and has a mortality rate of more than $50 \% .{ }^{(12)}$ Radiological findings are similar to acute respiratory distress syndrome and vary according to the phase of the disease. ${ }^{(13)} \mathrm{CT}$ is considered the gold standard in characterising ILD. The presence of new ground-glass opacities in a background of honeycombing suggests an acute exacerbation of ILD or superimposed infection. ${ }^{(14)}$ The mortality rate is more than $80 \%$ within one month of acute exacerbation. ${ }^{(14)}$

\section{Hypovolaemic shock}

Pleuropulmonary bleeding can be life-threatening, leading to hypovolaemic shock. In patients with haemoptysis, haemothorax can remain clinically undiagnosed or underestimated even when pulmonary sources of bleeding are suspected. Causes of nontraumatic haemothorax include spontaneous pneumothorax, coagulopathy, malignancy and, rarely,
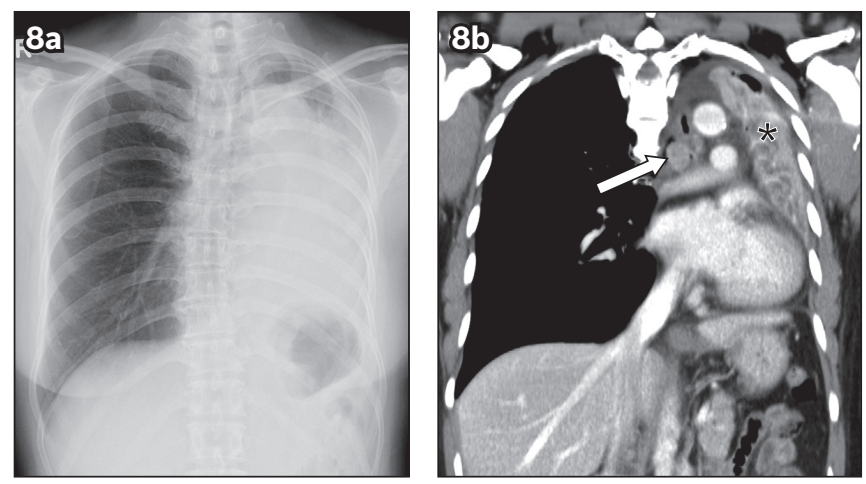

Fig. 8 Acute left lung collapse due to an endobronchial carcinoid tumour in the left main bronchus in a 51-year-old woman presenting to the emergency department with sudden onset dyspnoea. (a) Frontal chest radiograph shows an opaque left hemithorax with a mediastinum that has shifted to the left. (b) Coronal CT image shows an endobronchial mass (arrow) with collapse of the left lung $\left(^{*}\right)$

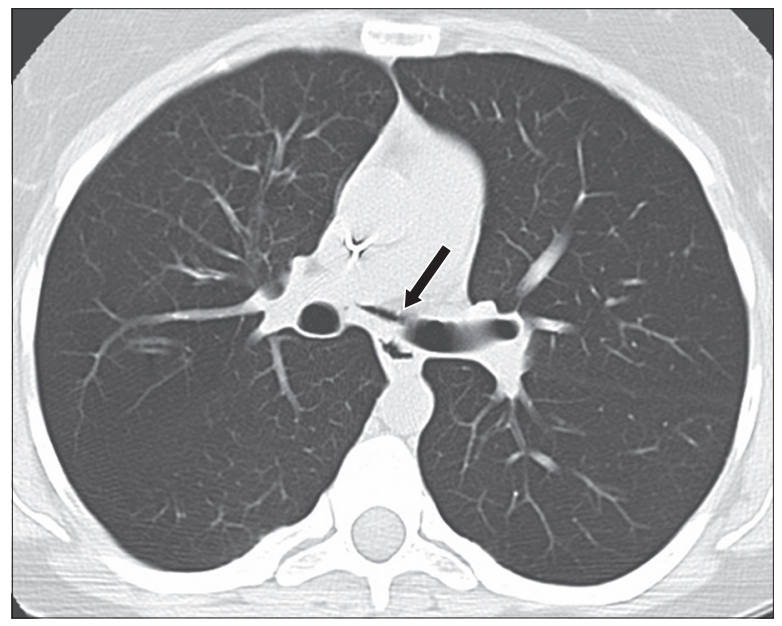

Fig. 9 Bronchial tear in a 34-year-old woman with acute exacerbation of steroid-resistant asthma. Axial CT image shows a defect in the medial wall of the left main bronchus (arrow) with small pneumomediastinum.

pulmonary arteriovenous malformation (PAVM). Imaging, particularly $\mathrm{CT}$, can demonstrate the presence of blood in the pleural cavity, which appears as high-density fluid. Contrastenhanced CT can identify the source of bleeding (Fig. 10) in cases of ruptured PAVM. ${ }^{(15)}$

\section{Haemoptysis}

Life-threatening haemoptysis in patients presenting to the ED could be due to malignancy, bronchiectasis, tuberculosis or 

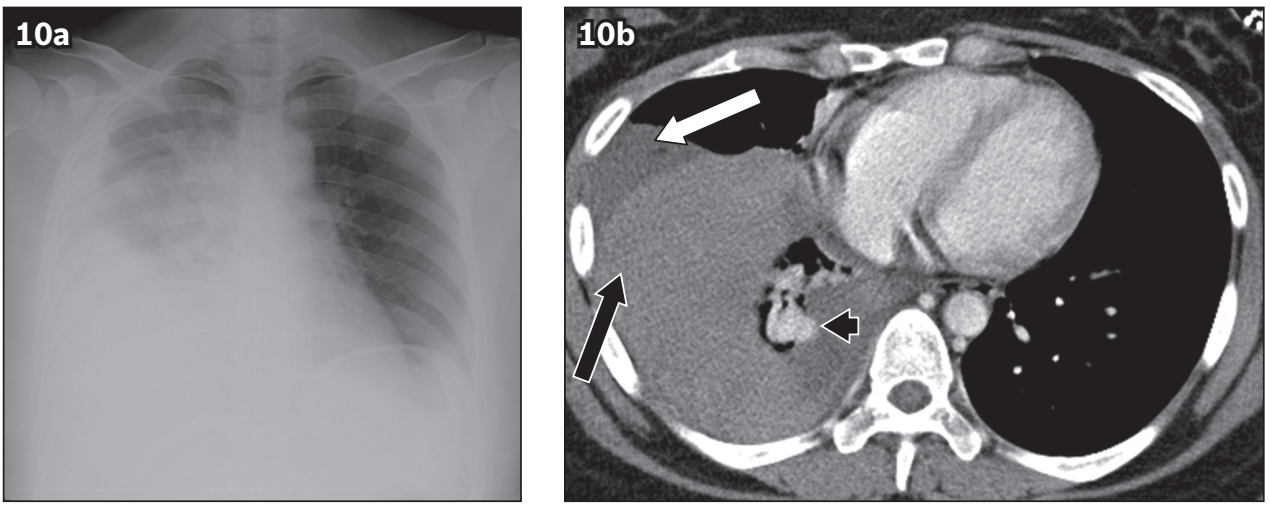

Fig. 10 Spontaneous haemothorax from an actively bleeding pulmonary arteriovenous malformation in a 26-year-old woman presenting to the emergency department with hypovolaemic shock, which was preceded by an episode of sharp right-sided chest pain. (a) Frontal chest radiograph shows a large amount of right-sided pleural fluid. (b) Axial CT image shows high-density dependent fluid (black arrow) and low-density nondependent fluid (white arrow) with an enhancing tortuous vascular malformation (arrowhead).
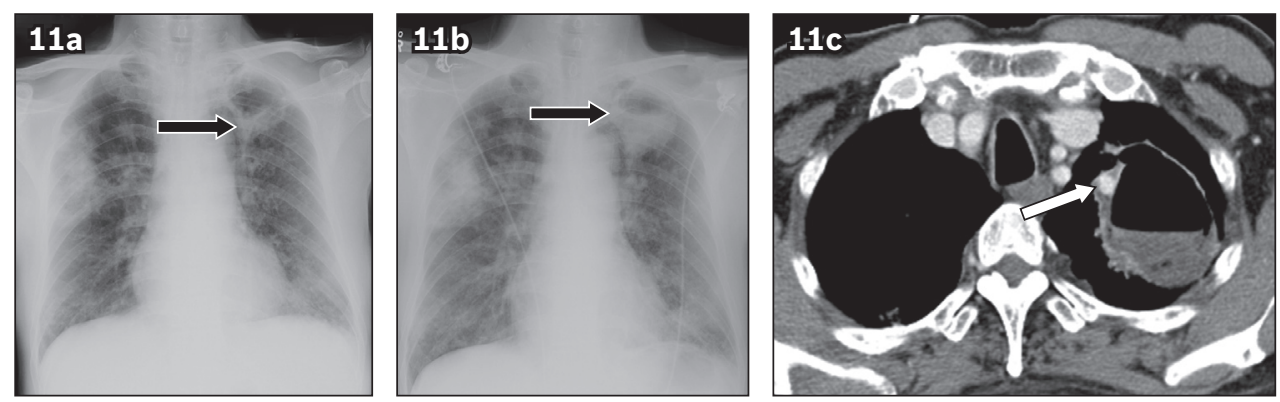

Fig. 11 Massive haemoptysis in a 54-year-old man. (a) Frontal chest radiograph on presentation to the emergency department shows right upper lung consolidation and a thick-walled cavity in the left upper lung (black arrow). (b) Frontal chest radiograph taken two hours later shows increased fluid level in the cavity (black arrow). (c) Axial CT image shows a small enhancing Rasmussen's aneurysm (white arrow) in the wall of the cavity. The fluid in the cavity is hyperdense, which suggests blood.
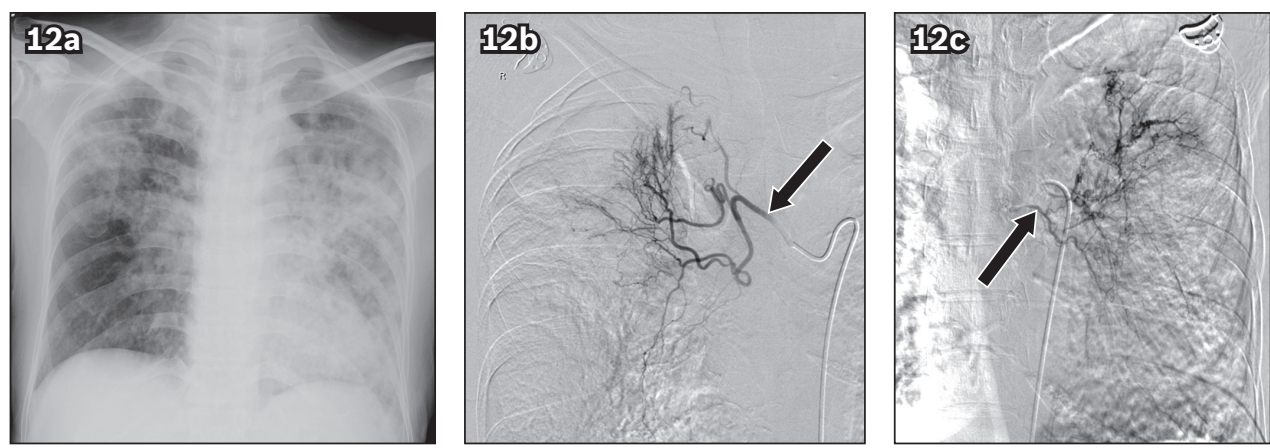

Fig. 12 Haemoptysis in a 51-year-old woman with pulmonary tuberculosis. (a) Frontal chest radiograph shows consolidation in both lungs. Angiograms of (b) the right bronchial artery (arrow) shows tortuous hypertrophied vessels in the right upper zone and (c) the left bronchial artery injection (arrow) also shows multiple tortuous hypertrophied branches that were embolised using polyvinyl alcohol particles.

pulmonary haemorrhage syndrome. The parenchymal changes associated with most of these cases can be identified on chest radiography. CT can identify lesions that are invisible on radiographs and provide more details about underlying pathology, such as demonstrating hypertrophied bronchial arteries, pseudoaneurysms and PAVMs. CT can also provide a road map for bronchoscopy or radiological intervention. ${ }^{(16,17)}$ In a majority of cases $(90 \%)$, bronchial arteries are the source of bleeding, while in a small number of cases (10\%), the culprit vessel originates from pulmonary circulation. Rare entities like Rasmussen's aneurysm, a mycotic aneurysm of the pulmonary artery (Fig. 11), can also be identified on CT. ${ }^{(18)}$ Transcatheter angiography and embolisation can be performed, if indicated (Fig. 12). The presence of a dilated, tortuous bronchial artery around the bronchus should be carefully evaluated in the absence of any pulmonary changes on $\mathrm{CT}$. It may be the only clue indicating Dieulafoy's disease of the bronchus, which is a rare vascular anomaly characterised by dilated vessels in the bronchial submucosa. ${ }^{(19)}$

Diffuse pulmonary haemorrhage or diffuse alveolar haemorrhage $(\mathrm{DAH})$ can present with life-threatening haemoptysis. 

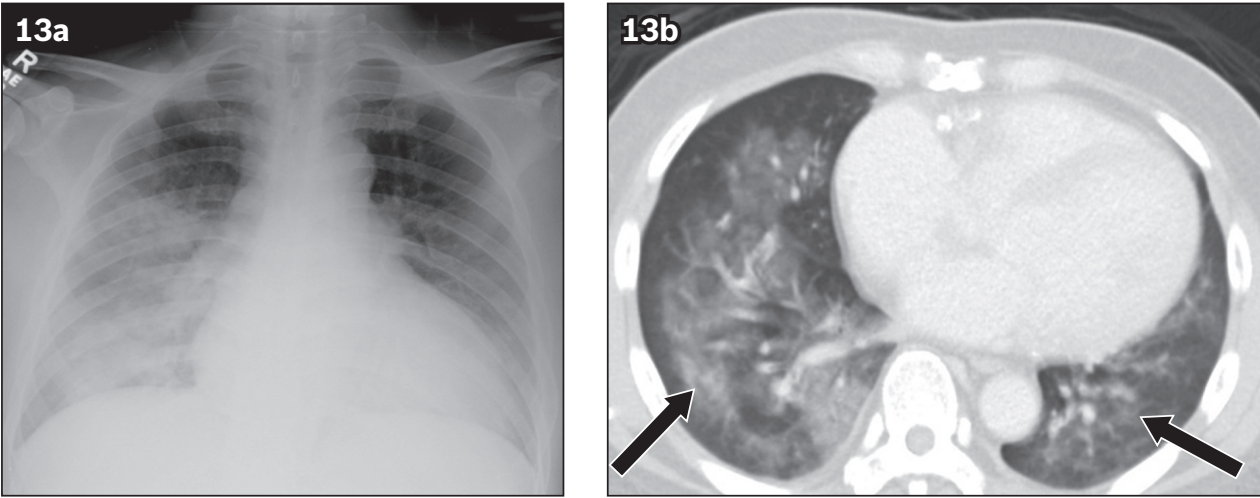

Fig. 13 Diffuse alveolar haemorrhage in a 61-year-old man on warfarin, presenting to the emergency department with haemoptysis. (a) Frontal chest radiograph shows asymmetric ground-glass opacities in the bilateral lower lungs. (b) Axial CT image shows ground-glass opacities (arrows) in both lungs, representing haemorrhage.
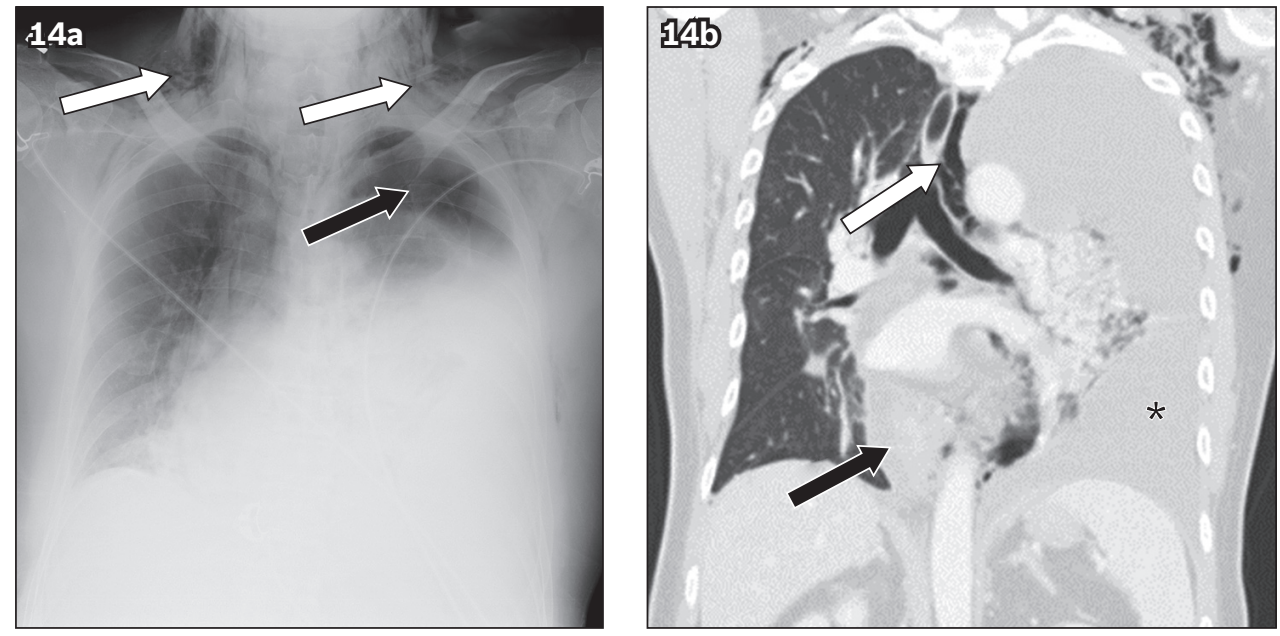

Fig. 14 Oesophageal tear in a 51-year-old man presenting to the emergency department with haematemesis preceded by retching. (a) Frontal chest radiograph shows a large left pleural effusion, left pneumothorax (black arrow) and chest wall emphysema (white arrows). (b) Coronal CT image shows a thickened lower oesophagus due to haematoma (black arrow), a large left pleural effusion $\left(^{*}\right)$ and pneumomediastinum (white arrow).

DAH is also associated with pulmonary infiltrates, dyspnoea and chronic anaemia. ${ }^{(20,21)}$ Its diagnosis is supported by the finding of haemorrhagic fluid in bronchoalveolar lavage. DAH is a clinical syndrome and includes a number of conditions, which are broadly divided into two categories: DAH with and without vasculitis. In clinical practice, the three most common causes of DAH are vasculitis, collagen vascular diseases and drugs (Fig. 13).

\section{Haematemesis}

Haematemesis is the result of acute upper gastrointestinal bleeding, commonly due to peptic ulcer disease, varices and oesophagitis. ${ }^{(22)}$ The role of imaging is limited and endoscopy remains the investigation of choice. Boerhaave syndrome is a rare but historically and clinically important condition that causes haemoptysis and can be suspected on imaging. ${ }^{(23)}$ In Boerhaave syndrome, a tear occurs in the posterior wall of the lower oesophagus, usually in the left wall. Classically, the patient has a history of overindulgence in food and drink, with violent vomiting and associated haemoptysis. Delayed diagnosis results in subcutaneous emphysema, chest pain and cardiovascular collapse. Chest radiography typically shows large pleural effusions, pneumomediastinum, pneumothorax and chest wall emphysema (Fig. 14). CT can demonstrate intramural haematoma in the oesophageal wall and perioesophageal air. Oesophagography may show leakage of contrast and oesophagopleural fistula. ${ }^{(24)}$

\section{Foreign body aspiration}

A foreign body lodged in the tracheobronchial tree can be lifethreatening due to airway obstruction (Fig. 15), while a foreign body ingested into the oesophagus (Fig. 16) can lead to serious complications such as haematemesis, oesophageal perforation and mediastinitis. Imaging is useful to localise radio-opaque foreign bodies and evaluate any associated complications.

\section{CONCLUSION}

Chest radiography and $\mathrm{CT}$ are useful tools for examining patients with emergency chest conditions. Normal chest radiography helps to rule out noncardiovascular emergencies. Abnormal or equivocal radiography findings can be due to various pathologies affecting the thoracic structures. Noncardiovascular acute conditions are less common and require a high index of suspicion on the part of the radiologist. Early diagnosis prevents a delay in definitive management in an often overcrowded ED. 

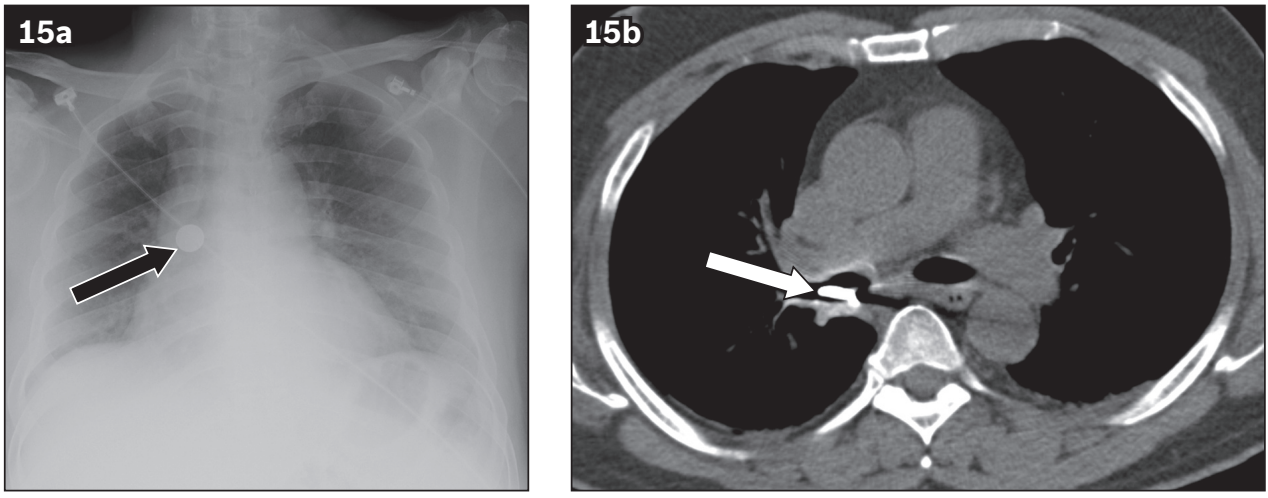

Fig. 15 Coin aspiration by a 51-year-old man. (a) Frontal chest radiograph shows the metallic foreign body projected over the right hilum (black arrow). (b) Axial CT image shows the coin lodged in the bronchus intermedius (white arrow).
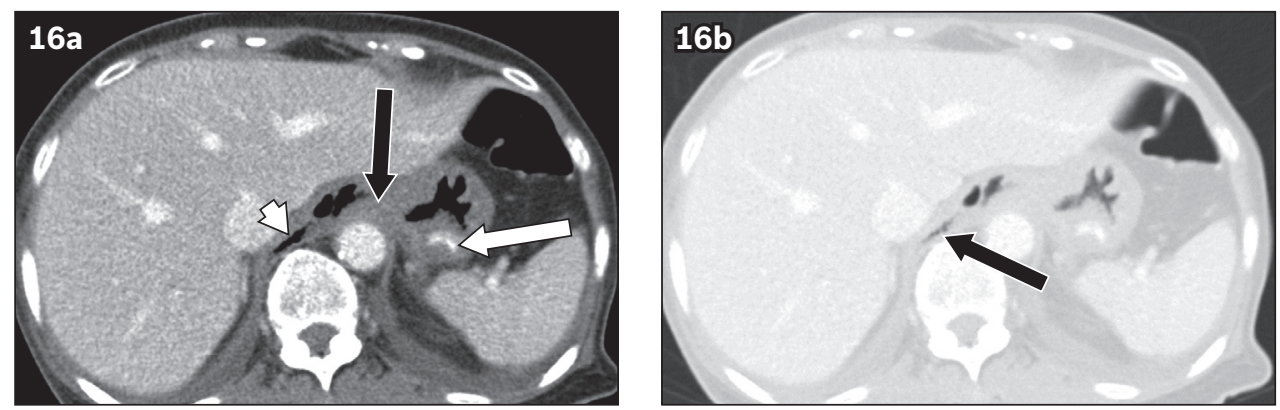

Fig. 16 Gastro-oesophageal perforation in a 28-year-old man after ingesting a chicken bone. (a) Axial CT image shows a curvilinear foreign body lodged in the gastric fundus (white arrow), the perforation site at the gastrooesophageal junction (black arrow) and free air (white arrowhead). (b) Axial CT image at the same level with a wider window setting shows pneumomediastinum (black arrow).

\section{REFERENCES}

1. Bhuiya FA, Pitts SR, McCaig LF. Emergency department visits for chest pain and abdominal pain: United States, 1999-2008. NCHS Data Brief 2010 43:1-8.

2. Luh SP. Review: Diagnosis and treatment of primary spontaneous pneumothorax. J Zhejiang Univ Sci B 2010; 11:735-44.

3. Amjadi K, Alvarez GG, Vanderhelst E, et al. The prevalence of blebs or bullae among young healthy adults: a thoracoscopic investigation. Chest 2007; 132:1140-5.

4. Hsu NY, Shih CS, Hsu CP, Chen PR. Spontaneous hemopneumothorax revisited: clinical approach and systemic review of the literature. Ann Thorac Surg 2005; 80:1859-63.

5. Macklin CC. Transport of air along sheaths of pulmonic blood vessels from alveoli to mediastinum: clinical implications. Arch Intern Med 1939; 64:913-26

6. Berkovich GY, Levine MS, Miller WT Jr. CT findings in patients with esophagitis. AJR Am J Roentgenol 2000; 175:1431-4.

7. Schmidt AJ, Stark P. Radiographic findings in Klebsiella (Friedlander's) pneumonia: the bulging fissure sign. Semin Respir Infect 1998; 13:80-2.

8. Kuhlman JE, Fishman EK, Teigen C. Pulmonary septic emboli: diagnosis with CT. Radiology 1990; 174:211-13.

9. Shamsuzzaman SM, Hashiguchi Y. Thoracic amebiasis. Clin Chest Med 2002; 23:479-92.

10. Woods AQ, Lynch DA. Asthma: an imaging update. Radiol Clin North Am 2009; 47:317-29.

11. American Thoracic Society; European Respiratory Society. American Thoracic Society/European Respiratory Society Internationa Multidisciplinary Consensus Classification of the Idiopathic Interstitial Pneumonias. This joint statement of the American Thoracic Society (ATS) and the European Respiratory Society (ERS) was adopted by the ATS board of directors, June 2001 and by the ERS Executive Committee, June 2001. Am J Respir Crit Care Med 2002; 165:277-304.

12. Mueller-Mang C, Grosse C, Schmid K, Stiebellehner L, Bankier AA. What every radiologist should know about idiopathic interstitial pneumonias. Radiographics 2007; 27:595-615.

13. Lynch DA, Travis WD, Müller NL, et al. Idiopathic interstitial pneumonias: CT features. Radiology 2005; 236:10-21

14. Kim DS, Park JH, Park BK, et al. Acute exacerbation of idiopathic pulmonary fibrosis: frequency and clinical features. Eur Respir J 2006; 27:143-50.

15. Berg AM, Amirbekian S, Mojibian $\mathrm{H}$, et al. Hemothorax due to rupture of pulmonary arteriovenous malformation: an interventional emergency. Chest 2010; 137:705-7.

16. Bruzzi JF, Rémy-Jardin M, Delhaye D, et al. Multi-detector row CT of hemoptysis. Radiographics 2006; 26:3-22.

17. Yoon W, Kim JK, Kim YH, Chung TW, Kang HK. Bronchial and nonbronchial systemic artery embolization for life-threatening hemoptysis: a comprehensive review. Radiographics 2002; 22:1395-409.

18. Khalil A, Parrot A, Nedelcu C, et al. Severe hemoptysis of pulmonary arterial origin: signs and role of multidetector row CT angiography. Chest 2008; 133:212-9

19. Parrot A, Antoine M, Khalil A, et al. Approach to diagnosis and pathological examination in bronchial Dieulafoy disease: a case series. Respir Res 2008; 9:58.

20. loachimescu OC, Stoller JK. Diffuse alveolar hemorrhage: diagnosing it and finding the cause. Cleve Clin J Med 2008; 75:258, 260, 264-5.

21. Primack SL, Miller RR, Müller NL. Diffuse pulmonary hemorrhage: clinical, pathologic, and imaging features. AJR Am J Roentgenol 1995; 164:295-300

22. Palmer K. Management of haematemesis and melaena. Postgrad Med J 2004; 80:399-404.

23. Derbes VJ, Mitchell RE Jr. Hermann Boerhaave's Atrocis, nec descripti prius, morbi historia, the first translation of the classic case report of rupture of the esophagus, with annotations. Bull Med Libr Assoc 1955; 43:217-240.

24. Ghanem N, Altehoefer C, Springer O, et al. Radiological findings in Boerhaave's syndrome. Emerg Radiol 2003; 10:8-13. 


\section{SINGAPORE MEDICAL COUNCIL CATEGORY 3B CME PROGRAMME} (Code SMJ 201511B)

Question 1. The Macklin effect explains:
(a) Spontaneous pneumothorax.
(b) Spontaneous pneumomediastinum.
(c) Spontaneous haemopneumothorax.
(d) Boerhaave syndrome.

Question 2. The mechanisms postulated for spontaneous haemopneumothorax are:
(a) Torn adhesion between the parietal and visceral pleura.
(b) Tear in a small noncontractile vessel on the parietal pleura.
(c) Rupture of a vascularised bulla.
(d) Dissection in the intercostal artery.

Question 3. Are the following statements true or false?
(a) The pulmonary artery is the usual source of bleeding in cases of haemoptysis.
(b) Rasmussen's aneurysm is a type of mycotic aneurysm.
(c) Diffuse alveolar haemorrhage can be seen in collagen vascular disease.
(d) Chest radiography can show parenchymal changes that account for haemoptysis.

Question 4. The bulging fissure sign is present in:
(a) Bronchogenic carcinoma.
(b) Boerhaave syndrome.
(c) Klebsiella pneumonia.
(d) Pulmonary arteriovenous malformation.

Question 5. The following complication(s) can be seen in asthmatic patients:
(a) Pneumothorax.
(b) Lobar collapse.
(c) Allergic bronchopulmonary mycosis.
(d) Eosinophilic pneumonia.

\footnotetext{
Doctor's particulars:

Name in full

MCR number

Specialty:

Email address

\section{SUBMISSION INSTRUCTIONS:}

(1) Log on at the SMJ website: http://www.sma.org.sg/publications/smjcurrentissue.aspx and select the appropriate set of questions. (2) Provide your name, email address and MCR number. (3) Select your answers and click "Submit".

\section{RESULTS:}

(1) Answers will be published in the SMJ January 2016 issue. (2) The MCR numbers of successful candidates will be posted online at the SMJ website by 4 January 2016 . (3) Passing mark is $60 \%$. No mark will be deducted for incorrect answers. (4) The SMJ editorial office will submit the list of successful candidates to the Singapore Medical Council. (5) One CME point is awarded for successful candidates.

Deadline for submission: (November 2015 SMJ 3B CME programme): 12 noon, 28 December 2015.
} 